\title{
On the use of aeromagnetism for geological interpretation part I: comparison of scalar and vector magnetometers for aeromagnetic surveys and an equivalent source interpolator for combining, gridding and transform fixed altitude and draping datasets
}

\section{B. Gavazzi ${ }^{1}$, L. Bertrand ${ }^{2}$, M. Munschy ${ }^{1}$, J. Mercier de Lépinay ${ }^{1}$, M. Diraison ${ }^{2}$, and Y. Géraud $^{2}$}

${ }^{1}$ Institut de Physique du Globe de Strasbourg (IPGS) ; UMR 7516 ; Université de Strasbourg/EOST, CNRS ; 1 rue Blessig, F-67084 Strasbourg Cedex, France.

${ }^{2}$ Université de Lorraine, CNRS, GeoRessources, F-54000 Nancy, France.

Corresponding author: Bruno Gavazzi (bgavazzi@unistra.fr)

Key Points:

- Total magnetic intensity surveys conducted with vector magnetometer systems offer the same data quality as with industrial scalar systems

- The use of potential field transforms on datasets that drape the topography can lead to significant errors

- An equivalent source interpolator allows merging different magnetic surveys and computes potential field transforms of draping dataset differences between this version and the Version of Record. Please cite this article as doi: 10.1029/2019JB018870 


\begin{abstract}
Airborne magnetic surveys are common in geosciences. High precision surveys are usually performed at a low altitude, draping the topography, using scalar absolute magnetometers and active compensation systems to correct the disturbances generated by the carrier. Another solution is to measure the total magnetic intensity using vector magnetometers, which allows compensation for aircraft fields to be calculated directly, without compensation systems. This study uses data of an industrial airborne survey and of a vector magnetometer survey with a small overlapping area in the Vosges (France) to compare both datasets and shows that both solutions provide results of the same overall precision. This paper also addresses the issue of combining fixed altitude and draped level datasets as well as topographic effect management by proposing an equivalent source interpolator based on a set of simple dipole sources. A synthetic case shows that the equivalent source interpolator allows to compute grids of the magnetic anomaly as well as potential field transforms from a draped dataset with an error level a few orders of magnitude lower than could be obtained with minimum curvature, a classicaly applied interpolator. The interpolator is also tested on a case study in the Vosges massif of which geological interpretation is presented in Bertrand et al. (2020). The results confirm the observations made on the synthetic case and show that the equivalent source interpolator is more tolerant to navigation errors (altitude) than minimum curvature and allows more rigorous computation of potential field transforms of draped datasets.
\end{abstract}

\title{
1 Introduction
}

Airborne magnetic surveys are performed at different scales and for various applications in geosciences. They can be used to study heterogeneities linked to contrasts of magnetization within the subsurface, such as igneous bodies or structural features (Nabighian et al., 2005). Airborne surveys are conducted with highly sensitive $(<0.01 \mathrm{nT})$ scalar magnetometers, usually alkali (cesium or potassium) vapor optically pumped magnetometers, mounted in a stinger rigidly mounted on an aircraft or a helicopter. A compensation unit is added to the system to correct for the magnetic field due to the aircraft on the sensor. It works in two steps (Coyle et al., 2014). First the magnetic effects of the carrier on the three components of the magnetic field are measured with a fluxgate three-component magnetometer during a calibration flight with \pm 10 degree rolls, \pm 5 degree pitches and \pm 5 degree yaws. The output is then used during the survey to compute real time corrections according to the orientation of the carrier, thus reducing heading and maneuvering errors on the scalar magnetometer used for surveying. In good conditions, heading and maneuvering errors are reduced down to \pm 0.25 and $\pm 0.15 \mathrm{nT}$ respectively (Horsfall, 1997; Luyendyk, 1997), i.e. a $0.8 \mathrm{nT}$ maximal absolute error, but a total error up to $2.0 \mathrm{nT}$ is usually accepted (Coyle et al., 2014). Another approach is to use fluxgate three-component magnetometers for measuring the earth's magnetic field. Almost abandoned for surveying in the 1960s due to a lack of precision, their use is nowadays usually limited to compensation units. However, improvements in calibration methods since the 1990s make the use of fluxgate magnetometers for surveying interesting again. The measurement of the three components of the magnetic field allows calibration and compensation of the system without a compensation unit by performing a simple procedure at the beginning of each flight. The intensity of the magnetic field, i.e. similar to the output of a scalar magnetometer, can then be computed without the need of precise orientation of the sensors. Indeed, Olsen et al. (2003) showed that such procedure, called a scalar calibration, allows to compute accurately enough the sensitivitiy, non-orthogonality and offset errors of each component of a fuxgate magnetometer and Munschy et al. (2007) showed that such procedure allows to compensate for the remanent and induced magnetization of the aircraft, all without the need of a 
procedure prior to the flight. The final root-mean-square (RMS) error is then less than $2 \mathrm{nT}$ at $25 \mathrm{~Hz}$ and corresponds mainly to white noise due to the digitization process. Gavazzi et al. (2017) showed that a calibrated fluxgate magnetometer mounted on a backpack for ground surveys has a RMS error of about $0.3 \mathrm{nT}$ which is in the same range as the expected error of a surveying device using absolute magnetometers and a compensation unit $(<0.8 \mathrm{nT}$ according to Coyle et al., 2014). Munschy and Fleury (2011) showed that this range of RMS error can be obtained in airborne surveys regardless of the level of magnetization of the carrier. Thus, surveying devices using the norm given by three-component fluxgate magnetometers should achieve the same range of precision as scalar magnetometers for geophysical applications. A difference between the two kinds of sensors can be found in the linear time-related drift of which only fluxgates are subjected to, but this is not an issue as it can be corrected through a good tie-line management (Coyle et al., 2014). This is why examples of fluxgates commercially used on aircrafts for surveying have been seen for a few years (e.g. Ameglio et al., 2011). In addition, their use could increase in the future as they can be potentially used with any vehicle, such as ultra-light aircraft, drones or any all-terrain vehicles due to their small weight and size as well as low power consumption (Gavazzi et al., 2016).

Regardless of the choice of sensors, magnetic data sampling density in airborne surveys is always greater along the profiles than across for economic reasons. Data are then recalculated on a regular grid using an interpolator in order to obtain maps of the anomaly and to allow the use of potential field transforms calculated in the spectral domain. Different interpolators exist, usually assuming the signal to be a smooth function, such as the minimum curvature (Briggs, 1974), one of the most common techniques in use. Such method consists of defining a thin elastic plate passing through the data points with as minimum bending as possible and is very efficient for fixed altitude surveys. However, it is sometimes better to minimize variation of distance to source by flying magnetic profiles that drape the topography over rugged terrain (Grauch \& Campbell, 1984). In this case, the use of potential field transforms in the spectral domain can induce errors because flatness of the grid is required for validity of Fourier-based processing.

This paper aims to offer a comparison of data quality between scalar and vector magnetometer airborne systems, as well as to propose an interpolator that does not require smoothness of data for combining, gridding and transforming data from a fixed altitude and a draped survey performed in the Northern Vosges, France.

\subsection{Survey area}

The studied area in the Vosges Mountains is a part of the shoulders of the Upper Rhine Graben (URG), where several deep drilling projects for heat and/or electricity production from natural hot hydrothermal fluids are being carried out (Boissavy et al., 2016). Due to the need of high temperatures $\left(>150^{\circ} \mathrm{C}\right)$ for these projects, the drilling targets are mainly located in the basement rocks of the graben at great depth $(2-4 \mathrm{~km})$. Theses rocks are commonly metamorphic or magmatic with very large petrographic variations and complex structural features derived from the geological history predating the graben history. Thus, the knowledge of the structural features controlling the fluid flows and the reservoir properties of these rocks are key information for the feasibility assessment of the projects, depending on the balance between production capacities and drilling costs.

In the Northern Vosges, previous terrestrial magnetic and gravimetric data have demonstrated that the basement rocks of the central URG are outcropping at the graben shoulders in the Northern Vosges Mountains (Figure 1). The area is therefore used to enhance the knowledge of the structures and petrographic variations within the basement rocks, and to 
enable more truthful extrapolation into the graben below the sedimentary cover (Bertrand et al., 2018).

With this aim, two magnetic surveys have been conducted in 2008 and 2015 with different objectives. The first one was conducted at a fixed altitude of $900 \mathrm{~m}$ above sea level with fluxgate magnetometers in order to study deep structures and lithology. The second one was conducted draping topography at a height of $300 \mathrm{~m}$ above ground with a scalar magnetometer in order to study also shallower units for industrial applications. Both surveys are overlapping over a small portion of the whole area (Figure 1). The whole area is characterized by rugged terrain, especially in the second survey with altitude variations exceeding $1000 \mathrm{~m}$.

\section{Comparison between scalar and vector magnetometer surveys}

For each survey, data are processed through the following steps

- For the fluxgate magnetometer survey the magnetic fields due to the carrier are corrected. For the scalar magnetometer survey these effects are compensated in real time by a compensation unit.

- Time-induced variations from external sources are removed using data from the Intermagnet observatory of Chambon-La-Forêt situated approximately $375 \mathrm{~km}$ westwards.

- The total magnetic field anomaly is obtained by subtracting the regional magnetic field using the IGRF 12 model (Thébault et al., 2015).

- Residual leveling effects and drifts are corrected by minimization of the differences by a constant value at the crossing points between profiles and tie-lines. As a too large difference in altitude at the crossing points might lead to errors in the correction due to different distances to the sources (Coyle et al., 2014) only the crossing points meeting an altitude difference criterion of less than $20 \mathrm{~m}$ are taken into account.

The quality of each dataset is assessed by checking the residual differences at the crossing points as they depend on any imprecision that may have occurred during the data acquisition, such as positioning or magnetometer sensitivity issues, as well as during the data processing, such as compensation or other corrections.

\subsection{Fixed altitude survey using vector magnetometers}

The first survey was conducted in 2008 by the Institut de Physique du Globe de Strasbourg with fluxgate vector magnetometers (Bartington Mag-03 MC) mounted at each end of the wings of a Maule MX7 airplane (Bouiflane, 2008; Munschy \& Fleury, 2011). The survey covers an area of $20 \times 30 \mathrm{~km}$ with N-S profiles acquired at a fixed altitude of $900 \mathrm{~m}$ above sea level. The speed of the aircraft along profiles is approximately $175 \mathrm{~km} / \mathrm{h}$ and the data are sampled at $27 \mathrm{~Hz}$, i.e. one measurement every $1.8 \mathrm{~m}$ along each profile. Line spacing is $500 \mathrm{~m}$ and tie-line spacing $2000-3000 \mathrm{~m}$. Calibration and compensation procedures are done by performing an eight shaped pattern with varying yaw, pitch and roll of the aircraft over a magnetically quiet area. After computation, the RMS error decreases from about 240 $\mathrm{nT}$ to $2 \mathrm{nT}$ (Figure 2). Time-induced variations are corrected and the regional field is removed. 49 out of 411 (12\%) crossing points meet the $20 \mathrm{~m}$ tolerance criterion. The low proportion of crossing points meeting the criterion is due to an error of navigation during some of the flights, which were set at an altitude of $1000 \mathrm{~m}$. The selected points are used for 
correction of leveling effects. The data quality can then be assessed by checking the residual differences at the crossing points after all processes: the standard deviation at the 49 crossing points is $1.3 \mathrm{nT}$.

\subsection{Draping survey using scalar magnetometers}

The second survey was flown in 2015 by Geophysics GPR International Inc. for Fonroche Géothermie with a cesium scalar magnetometer (Geometrics) mounted on an AS 350 Ecureuil Helicopter. The manufacturer gives a sensitivity of $0.003 \mathrm{nT}$ for the magnetometer. The survey covers an area of $28 \times 33 \mathrm{~km}$ with profiles acquired draping the topography at a constant height of $300 \mathrm{~m}$. Therefore, the altitude of flight varies between 440 and $1400 \mathrm{~m}$ above sea level. The speed of the helicopter is approximately $125 \mathrm{~km} / \mathrm{h}$ and the data are sampled at $10 \mathrm{~Hz}$, i.e. one measurement every $3.5 \mathrm{~m}$ along each profile. Line spacing is $400 \mathrm{~m}$ and tie-line spacing $4000 \mathrm{~m}$. Compensation is performed in real time with an AARC500 unit from RMS Instruments. Time induced variations are corrected and the regional field is removed. 221 out of $558(40 \%)$ crossing points meet the $20 \mathrm{~m}$ tolerance criterion. After correction of the levelling effects the data quality is assessed by checking the residual differences at the crossing points: the standard deviation at the 221 crossing points is $0.8 \mathrm{nT}$.

\subsection{Comparison}

An apparent difference between the measurements of each device is the noise level which is shown in Figure 3: data measured with a vector magnetometer show noise with a greater amplitude than data measured with a scalar magnetometer. However, in both cases this noise is mainly gaussian and with low intensities (mostly $<0.5 \mathrm{nT}$ ). More importantly, the noise is of higher frequencies than the targeted signal and is mostly eliminated through the gridding computation. An appropriate way to assess the overall quality of the measurements is to check the differences at the crossing points after the entire computational procedure for each survey. The result provides a good indicator of the datasets' overall quality as it is sensitive to any imprecision that may have occured during the measurement and computation processes, such as positioning or magnetic compensation of the measuring device and carrier. Thus we can observe that even though scalar cesium magnetometers are very precise sensors with a sensitivity of $0.003 \mathrm{nT}$, the corresponding survey has an overall residual RMS error of $0.8 \mathrm{nT}$ due to other sources of imprecision. Similarly, the survey flown with vector magnetometers shows an overall residual RMS error of $1.3 \mathrm{nT}$. According to these results, both types of equipment offer the same order of precision, and are suited for geophysical applications by having a precision better than 2 nT (Coyle et al., 2014). In conclusion, this comparison shows that the industrial cesium based device and the lighter and more costeffective fluxgate device offer a same overall precision of 1-2 nT which is suitable for geoscience applications. A graphical illustration of the similarities of the quality of the dataset can be found in Gavazzi et al. (2019) in which the overlapping part of the dataset is shown to present similar results.

\section{Equivalent source for gridding}

\subsection{Definition}

The equivalent source principle, first proposed by Dampney (1969), consists of using the non-uniqueness of the solution in potential field problems to our advantage: the different 
parameters of sources are not decipherable from data without a-priori constraints. It is therefore possible to find a set of simple sources that explain the data, 'the equivalent sources', from which new data points can be calculated. The strong advantage of this method is that it takes into account that the anomaly function is harmonic and does not require a particular distribution of the initial data points. Different methods of calculation of the equivalent sources have been developed for different applications such as continuation (Pilkington \& Boulanger, 2017) and reduction to the pole (e.g. Guspi \& Novara, 2009; Li et al., 2014 or Silva, 1986), but it is not commonly used as an interpolator for gridding. Different strategies have nonetheless been developed with different advantages and disadvantages. Dampney (1969) places a source under each data point on a horizontal plane at a fixed depth. Bhattacharrya \& Chan (1977) define a set of equivalent sources on the observation surface. Cordell (1992) proposes to reduce the number of sources by proceeding iteratively for each data point, starting with the most anomalous one, and combining the superimposing sources. Pilkington \& Urquhart (1990) place sources on a surface that mirrors the topography of the survey. Mendonça and Silva (1994) split the data into subsets and place a number of equivalent sources, greater than the number of data, at a fixed depth. The solution of the underdetermined problem with the minimum euclidean norm is then chosen. Barnes \& Barraud (2012) define sources as rectangular prisms with varying bottom depth. Most of these strategies are compared in Pilkington \& Boulanger (2017). Here, we propose an approach in which the aim is not to model coherently the subsurface but to use a set of simple sources that can be easily computed to be used only as an interpolator. We chose for the equivalent source a sphere of unit radius with only induced magnetization. In a Cartesian coordinates system with the $x$ axis to the north, the $y$ axis to the east and the $z$ axis downward, the magnetic potential $U(x, y, z)$ of such a source placed at $\left(x_{0}, y_{0}, z_{0}\right)$ can be written

$$
U(x, y, z)=C \frac{M \cdot r}{r^{3}},
$$

where $C=\frac{10^{9} \mu_{0} V}{4 \pi}=\frac{400 \pi}{3}$ is a constant variable containing the vacuum magnetic permeability $\mu_{0}$, the volume of the sphere $V$ and the conversion factor $10^{9}$ to obtain a potential in nT.m in SI (Tm), $\vec{M}\left(M_{x}, M_{y}, M_{z}\right)$ is the total magnetization vector and $r\left(r_{x}, r_{y}, r_{z}\right)$ is the distance vector between the source and the observation point. Assuming only induced magnetization $\vec{M}\left(M_{x}, M_{y}, M_{z}\right)$ can be decomposed as:

$$
\begin{gathered}
M x=M \cos (D) \cos (I)=M \alpha, \\
M_{y}=M \sin (D) \cos (I)=M \beta, \\
M_{z}=M \sin (I)=M \gamma,
\end{gathered}
$$

where $M$ is the intensity of magnetization, $D$ and $I$ are the magnetic declination and inclination of the regional magnetic field. Then equation (1) can be rewritten

$$
U(x, y, z)=C M \frac{1}{r^{3}}\left(\alpha r_{x}+\beta r_{y}+\gamma r_{z}\right),
$$

from which the magnetic field $\vec{B}(x, y, z)$ can be derived as 


$$
\vec{B}(x, y, z)=C M \frac{1}{r^{5}} \begin{gathered}
B_{x} \\
\left(B_{y}\right) \\
B_{z}
\end{gathered}
$$

where

$$
\begin{aligned}
& B_{x}=\alpha r^{2}-3 r_{x}\left(\alpha r_{x}+\beta r_{y}+\gamma r_{z}\right), \\
& B_{y}=\beta r^{2}-3 r_{y}\left(\alpha r_{x}+\beta r_{y}+\gamma r_{z}\right), \\
& B_{z}=\gamma r^{2}-3 r_{z}\left(\alpha r_{x}+\beta r_{y}+\gamma r_{z}\right) .
\end{aligned}
$$

As $A(x, y, z)$ the anomalous intensity of the magnetic field is, to a good approximation, the projection of $\vec{B}$ onto the regional field in the usual case for which the anomalous field is small compared to the regional geomagnetic field, it can be written

$$
A(x, y, z)=C M \frac{1}{r^{5}}\left(\alpha B_{x}+\beta B_{y}+\gamma B_{z}\right) .
$$

Now the equivalent-sources principle can be written as the following linear problem

$$
d=G p \text {, }
$$

where $d$ is a vector with $N\left(x_{n}, y_{n}, z_{n}\right)$ observations (magnetic anomaly), $p$ is a vector with $M\left(x_{m}, y_{m}, z_{m}\right)$ sources with unknown parameters (magnetization intensity) and $G$ is a $N \times M$ matrix that maps the effect of each source of unit value on every data point. Thus the element $G_{n, m}$ of $G$ can be written

$$
G_{n, m}=C \frac{1}{r^{5}}\left(\alpha B_{x}+\beta B_{y}+\gamma B_{z}\right)
$$

The aim now is to find a minimum number of sources that can offer a satisfactory fit to the data. Sources could be placed under every data point, but it can be considered redundant, as the distance between samples will be shorter along profiles than across. As the distance between profiles is a limiting factor, the sampling distance between sources along profiles could be set to be the same as the distance between profiles. This choice provides the shortest computation time but does not benefit from the fact that sources will be better constrained along profiles than across. In the end, any sampling distance along profiles between these two extrema could be chosen. The presence of aliasing effects depends on the ratio of distance from data points to sources and between data points. Dampney (1969) estimates empirically that the distance to the sources should be between 2.5 and 6 times the spacing distance between data points for gravimetric surveys. The 2.5 lower limit is often used in gravimetric as well as magnetometric surveys, seemingly at face value without further discussion. Here we choose to keep the distance between a source and the data point above it constant, at 5 times the spacing distance, according to best fits of the synthetic case described in the following section.

\subsection{Synthetic case}

To test our approach, we propose a simple synthetic model. We place 15 simple spheres of unit volume at depths ranging from 200 to 300 meters and magnetization intensities ranging from 100 to $1000 \mathrm{~A} / \mathrm{m}$ (Figure 4). The intensities are set at such values in order to approximate, at a sufficient distance, structures with a more complex geometry, higher volume and smaller intensity. Synthetic flight lines are simulated with a varying altitude ranging between 0 and $75 \mathrm{~m}$. A spacing of $50 \mathrm{~m}$ between profiles and a sampling distance of $1 \mathrm{~m}$ along the profiles are set (Figure 4). For the equivalent source method, 
sources are placed under every 25 data points along the profiles. Thus 841 equivalent sources are set for 21021 data points. The inclination and declination of the magnetic field are arbitrary set to local values of $64.3^{\circ}$ and $1.7^{\circ}$ respectively. We then compare gridding results from equivalent source and minimum curvature methods to the 'true' values of the magnetic anomaly obtained by direct modeling from the synthetic model. The node spacing of the grid is $25 \mathrm{~m}$, i.e. half the profile spacing as is the common practice in aeromagnetic data processing. The equivalent source method allows to compute grids at a fixed altitude or varying flight level while minimum curvature provides a unique solution. Reduction to the pole (RTP) is also computed for each grid to illustrate the effects of each gridding method on a potential field transform. It is normally calculated using Fourier transforms but can also be computed directly from the equivalent sources through a simple direct calculation (Blakely, 1995) .

Graphic comparisons and associated errors between the model and grids obtained through equivalent source and minimum curvature are shown in Figure 5. For the magnetic anomaly grids, both minimum curvature and equivalent source methods show a mean error with the model of $0.1 \%$ or less, which we deem acceptable. However, the results of the equivalent source method show a mean error three orders of magnitude lower than those obtained with minimum curvature. For the RTP, the differences are more significant and could lead to errors of interpretation. The RTP calculated from the grid computed with minimum curvature shows an error of $6.14 \%$ with the true RTP at a fixed level and $17.5 \%$ with the true RTP at the varying flight level (i.e. draping). The RTP computed from the equivalent sources is the one that provides a better fit, as the mean error is in the order of $0.001 \%$ or less. According to these results, both the equivalent source and minimum curvature can be considered as a valid methods for gridding data acquired at any level as they show both low error levels $(<0.1 \%)$. However, computation of potential field transforms such as RTP through direct calculation from equivalent sources show better results (errors $<0.01 \%$ ) than through Fourier transforms from minimun curvature (error $>5 \%$ ). Therefore equivalent sources seems to be a better solution than minimum curvature for potential field transforms such as RTP for data that are not acquired at a constant altitude.

In addition, we tested the behavior of both minimum curvature and equivalent sources in the presence of noise. Indeed, results from aeromagnetic surveys might present noise in the form of leveling effects due to navigation or compensation discrepancies. To simulate this noise, every other simulated profile is displaced by $20 \mathrm{~m}$ in $\mathrm{x}, \mathrm{y}$ and $\mathrm{z}$ directions, and the magnetic anomaly at grid level 0 , which corresponds to the lowest altitude in the simulated flight lines, is computed through both methods (Figure 6). White Gaussian noise due to the sensors and digitization processes is not taken into account as it is usually eliminated through the gridding step. Figure 6 shows that only the map computed with minimum curvature displays leveling effects. Therefore equivalent sources seems to be a better solution than minimum curvature for the interpolation of datasets with noise.

\section{Case study}

Equivalent source gridding is used on the data of both surveys. To reduce the computing time to a minimum, the distance between sources along profiles is set equal to the distance across profiles.

\subsection{Fixed altitude survey}

For the fixed altitude survey, equivalent sources are defined every $500 \mathrm{~m}$ along the profiles (i.e. equal the distance between the profiles). This requires 2241 sources. A grid with a spacing equal to half the profile spacing, i.e. $250 \mathrm{~m}$, is then computed with each gridding 
method. The result of equivalent source gridding at a fixed altitude of $900 \mathrm{~m}$ shows a strong reduction of the residual $\mathrm{N}-\mathrm{S}$ lines pattern seen in the result of the minimum curvature gridding (Figure 7). In addition, many of the anomalies seem more apparent with the equivalent source than with the minimum curvature, possibly due to the correction of navigation errors.

\subsection{Draping survey}

For the draped survey, equivalent sources are defined every $400 \mathrm{~m}$ along the profiles (i.e. equal to the distance between the profiles). It corresponds to 5375 sources. Differences of shapes and intensities between results can be observed (Figure 8). As expected from the results of the synthetic case, differences between draped grids obtained by minimum curvature and equivalent sources are not significant. This illustrates that for producing a map of the magnetic anomalies when the navigation is optimal and uniform (i.e. a constant distance to the ground of fixed altitude), the choice of processing algorithm is less relevant. Differences between fixed altitude $(1400 \mathrm{~m})$ computed from equivalent sources and draping grids are due to different distance to sources and illustrate how interpretation could differ between surveys with different navigation parameters. But more importantly in the case of a draping survey, equivalent sources allow the computation of potential field transforms taking into consideration the non-flatness of the dataset. It is possible to calculate a grid at any constant level from which transforms can be computed using a fast Fourier transform (FFT), or to use direct calculation in the spatial domain to obtain transforms on a flat or draped grid. An illustration of the calculation of reduction to the pole by direct calculation from equivalent sources compared to using FFT on a grid obtained through minimum curvature is shown in Figure 9. As predicted by the synthetic case, differences between the maps can be observed: the calculation of RTP from the grid obtained with minimum curvature does not fit any of the maps calculated from equivalent sources, neither on a surface draping the topography or flat level at $1400 \mathrm{~m}$ of altitude. This illustrates that that the computation of potential field transforms such as RTP from a grid obtained with minimum curvature from a non fixed level dataset can lead to error and should be used with caution. According to the synthetic case, computation from equivalent sources both at a draped and a fixed level are more accurate.

\subsection{Combination of datasets}

Both surveys can be studied separately, but combining them to have a large scale overview of all the area can be useful for interpretation. As both surveys were flown with different parameters a simple fusion would be difficult to interpret. The combination can be done through the same equivalent source process as for each survey in order to compute grids with controlled altitude (draping or fixed level). For the combination, equivalent sources are defined every $500 \mathrm{~m}$ along the profiles (i.e. equal to the maximal distance between the profiles). It corresponds to 8210 sources. The magnetic anomaly computed from those equivalent sources on a flat grid at $1400 \mathrm{~m}$ of altitude as well as on a grid draping the topography at a height of $200 \mathrm{~m}$ can be seen in Figure 10. Both kinds of grid can be used for different interpretations. Further interpretative methods (potential field transforms and direct modeling) are applied to the grid at a fixed altitude of $1400 \mathrm{~m}$ and discussed for a geological interpretation of the deep features of the area in Bertrand et al. (this issue). 


\section{Conclusions}

The comparison between the two aeromagnetic surveys shows that fluxgate threecomponent magnetometers can be used for aeromagnetic surveys. They provide the same level of accuracy as common industrial systems using scalar magnetometers without the need for a real time compensation unit. Additionally, their light weight and low power consumption open the possibility to use them on lighter carriers in the future. By reducing operation costs, the use of fluxgate magnetometers could increase the accessibility of aeromagnetic surveys in geosciences, especially in projects with low financial impact perspectives or without direct commercial interest.

Regarding gridding and combining datasets, the equivalent source method proves to be an excellent interpolator to take into account the variations of altitude of measurements due to navigation errors or survey settings. Minimum curvature and equivalent source give both accurate results when computing intensity anomalies of the Earth's magnetic field of draping dataset, but only equivalent source is able to correct the effects of navigation errors and to provide accurate potential field transforms of draping datasets for interpretation. As a result, an equivalent source method could be used to correct navigation errors, to combine efficiently datasets from surveys with different altitude settings and to provide potential field transforms of non-fixed level dataset with a greater accuracy than through the use of FFT computation from grids obtained with a minimum curvature method.

\section{Acknowledgments, Samples, and Data}

Some of the results presented in this paper rely on the data collected at Chambon-La-Forêt observatory. We thank Centre de Recherche en Astronomie, Astrophysique et Géophysique as well as Institut de Physique du Globe de Paris, for supporting its operation and INTERMAGNET for promoting high standards of magnetic observatory practice (www.intermagnet.org). The authors would also like to thank Fonroche Géothermie for providing the data of the survey carried out by GPR International. The data used in this paper are available in Bertrand et al (2019) and Gavazzi (2020). This article was greatly improved by the comments and corrections by David Clark, Mark J. Dekkers and an anonymous reviewer who we would like to thank.

\section{References}

Ameglio, L., Jacobs G., Von Ludwig, J., \& Munschy, M. (2011). GyroLAG - fluxgate magnetic total field, vectors, and tensors mapping. The Leading Edge, 30(6), 674-680, https://doi.org/10.1190/1.3599154

Barnes, G., \& Barraud, J. (2012) Imaging geologic surfaces by inverting gravity gradient data with depth horizons. Geophysics, 77(1), 1JF-Z19, https://doi.org/10.1190/geo2011$\underline{0149.1}$

Bertrand, L., Jussaume, J., Géraud, Y., Diraison, M., Damy, P-C., Navelot, V., \& Haffen, S. (2018). Structural heritage, reactivation and distribution of fault and fracture network in a rifting context: Case study of the western shoulder of the Upper Rhine Graben. Journal of Structural Geology, 108, 243-255, https://doi.org/10.1016/j.jsg.2017.09.006

Bertrand, L., Gavazzi, B., Mercier de Lépinay, J., Diraison, M., Géraud, Y., \& Munschy, M. (2019). Magnetic data grids of the Northern Vosges, surveys EOST2008 and GPR2015. Data set. Zenodo. https://doi.org/10.5281/zenodo.3520870 
Bhattacharyya, B. K., \& Chan, K. C. (1977). Reduction of magnetic and gravity data on arbitrary surfaces acquired in a region of high topographic relief. Geophysics, 42, 1411-1430, https://doi.org/10.1190/1.440802

Blakely, R. J. (1995). Potential theory in gravity and magnetic applications. Cambridge University Press. ISBN 0-521-41508-X

Boissavy, C., Rocher, P., Laplaige, P., \& Brange, C. (2016). Geothermal Energy Use, Country Update for France. European Geothermal Congress 2016 Strasbourg France, 1-19.

Bouiflane, M. (2008). Cartographies aéromagnétique et magnétique multi-échelles : étude structural d'une région du fossé rhénan. PhD Thesis Université Louis Pasteur, Strasbourg, France, 209 pages

Bourgeois, O., Ford, M., Diraison, M., Le Carlier de Veslud, C., Gerbault, M., Pik, R., et al. (2007). Separation of rifting lithospheric folding signatures in the NW Alpine foreland. International Journal of Earth Sciences, 96, 1003-1031, https://doi.org/10.1007/s00531-007-0202-2.

Briggs, I. C. (1974). Machine contouring using minimum curvature. Geophysics, 39(1), 3948, https://doi.org/10.1190/1.1440410

Cordell, L. (1992). A scattered equivalent-source method for interpolation and gridding of potential-field data in three dimensions. Geophysics, 57(4), 629-636, https://doi.org/10.1190/1.1443275

Coyle, M., Dumont, R., Keating, P., Kiss, F., \& Miles, W. (2014). Geological Survey of Canada aeromagnetic surveys: design, quality assurance, and data dissemination. Geological Survey of Canada, Open File 7660, 48 pages, https://doi.org/10.4095/295088

Dampney, C. N. G. (1969). The equivalent source technique. Geophysics, 34(1), 39-53, https://doi.org/10.1190/1.1439996

D'Errico, J. (2006). Surface Fitting using gridfit (https://www.mathworks.com/matlabcentral/fileexchange/8998-surface-fitting-usinggridfit), MATLAB Central File Exchange.

Edel, J. B., \& Schulmann, K. (2009). Geophysical constraints and model of the "Saxothuringian and Rhenohercynian subductions - magmatic arc system" in NE France and SW Germany. Bulletin de la Société géologique de France, 180(6), 545558, https://doi.org/10.2113/gssgfbull.180.6.545

Gavazzi, B. (2020). Synthetic model parameters for the comparison between an equivalent source and a minimum curvature interpolator of aeromagnetic data. Data set. Zenodo. https://doi.org/10.5281/zenodo.3706439

Gavazzi, B., Alkhatib-Alkontar, R., Munschy, M., Colin, F., \& Duvette, C. (2017). On the Use of Fluxgate 3-Axis Magnetometers in Archaeology: Application with a Multisensor Device on the Site of Qasr 'Allam in the Western Desert of Egypt. Archaeological Prospection, 24(1), 59-73, https://doi.org/10.1002/arp.1553

Gavazzi, B., Le Maire, P. Mercier de Lépinay, J., Calou, P. \& Munschy, M. (2019). Fluxgate three-component magnetometers for cost-effective ground, UAV and airborne magnetic surveys for industrial and academic geoscience applications and comparison 
with current industrial standards through case studies. Geomechanics for Energy and the Environment, 20, 100117, https://doi.org/10.1016/j.gete.2019.03.002

Gavazzi, B., Le Maire, P., Munschy, M., \& Dechamp, A. (2016). Fluxgate vector magnetometers: A multisensor device for ground, UAV, and airborne magnetic surveys. The Leading Edge, 35(9), 795-797, https://doi.org/10.1190/tle35090795.1

Grauch, V. J. S., \& Campbell, D. L. (1984). Does draping aeromagnetic data reduce terraininduced effects?. Geophysics, 49(1), 75-80, https://doi.org/10.1190/1.1441563

Guspi, F., \& Novara, I. (2009). Reduction to the pole and transformations of scattered magnetic data using Newtonian equivalent sources. Geophysics, 74(5), L67-L73, https://doi.org/10.1190/1.3170690

Horsfall, K. R. (1997). Airborne magnetic and gamma-ray data acquisition. AGSO Journal of Australian Geology and Geophysics, 17(2), 23-30.

Li, Y., Nabighian, M., \& Oldenburg, D. W. (2014). Using an equivalent source with positivity for low-latitude reduction to the pole without striation. Geophysics, 79(6), J81-J90, https://doi.org/10.1190/GEO2014-0134.1

Luyendyk, A. P. J. (1997). Processing of airborne magnetic data. AGSO Journal of Australian Geology and Geophysics, 17(2), 31-38.

Mendonça, A., \& Silva, J. B. C. (1994). The equivalent data concept applied to the interpolation of potential field data. Geophysics, 59(5), 722-732, https://doi.org/10.1190/1.1443630

Munschy, M., Boulanger, D., Ulrich, P., \& Bouiflane, M. (2007). Magnetic mapping for the detection and characterization of UXO: Use of multi-sensor fluxgate 3-axis magnetometers and methods of interpretation. Journal of Applied Geophysics, 61, 168-183, https://doi.org/10.1016/j.jappgeo.2006.06.004

Munschy, M., \& Fleury, S. (2011). Scalar, vector, tensor magnetic anomalies: measurement or computation?. Geophysical Prospecting, 59, 1035-1045, https://doi.org/10.1111/j.1365-2478.2011.01007.x

Nabighian, M. N., Grauch, V.J. S., Hansen, R. O., LaFehr, T. R., Li, Y., Peirce, J. W., et al. (2005). The historical development of the magnetic method in exploration. Geophysics, 70(6), 33ND-61ND, https://doi.org/10.1190/1.2133784

Olsen, N., Tøffner-Clausen, L., Sabaka, T.J., Brauer, P., Merayo, J.M.G., Jørgensen, et al. (2003). Calibration of the Ørsted vector magnetometer. Earth Planets Space, 55, 1118, https://doi.org/10.1186/BF03352458

Pilkington, M., \& Boulanger, O. (2017). Potential field continuation between arbitrary surfaces - Comparing methods. Geophysics, 82(3), J9-J25, https://doi.org/10.1190/GEO2016-0210.1

Pilkington, M., \& Urquhart, W. E. S. (1990). Reduction of potential field data to a horizontal plane. Geophysics, 55, 549-555, https://doi/10.1190/1.1442866

Silva, J. B. C. (1986). Reduction to the pole as an inverse problem and its application to lowlatitude anomalies. Geophysics, 51(2), 369-382, https://doi.org/10.1190/1.1442096

Thébault, E., Finlay, C. C., Beggan, C., Alken, P., Aubert, J., Barrois, O., et al. (2015), International Geomagnetic Reference Field: the 12th generation. Earth Planets Space, 67-79, https://doi.org/10.1186/s40623-015-0228-9. 


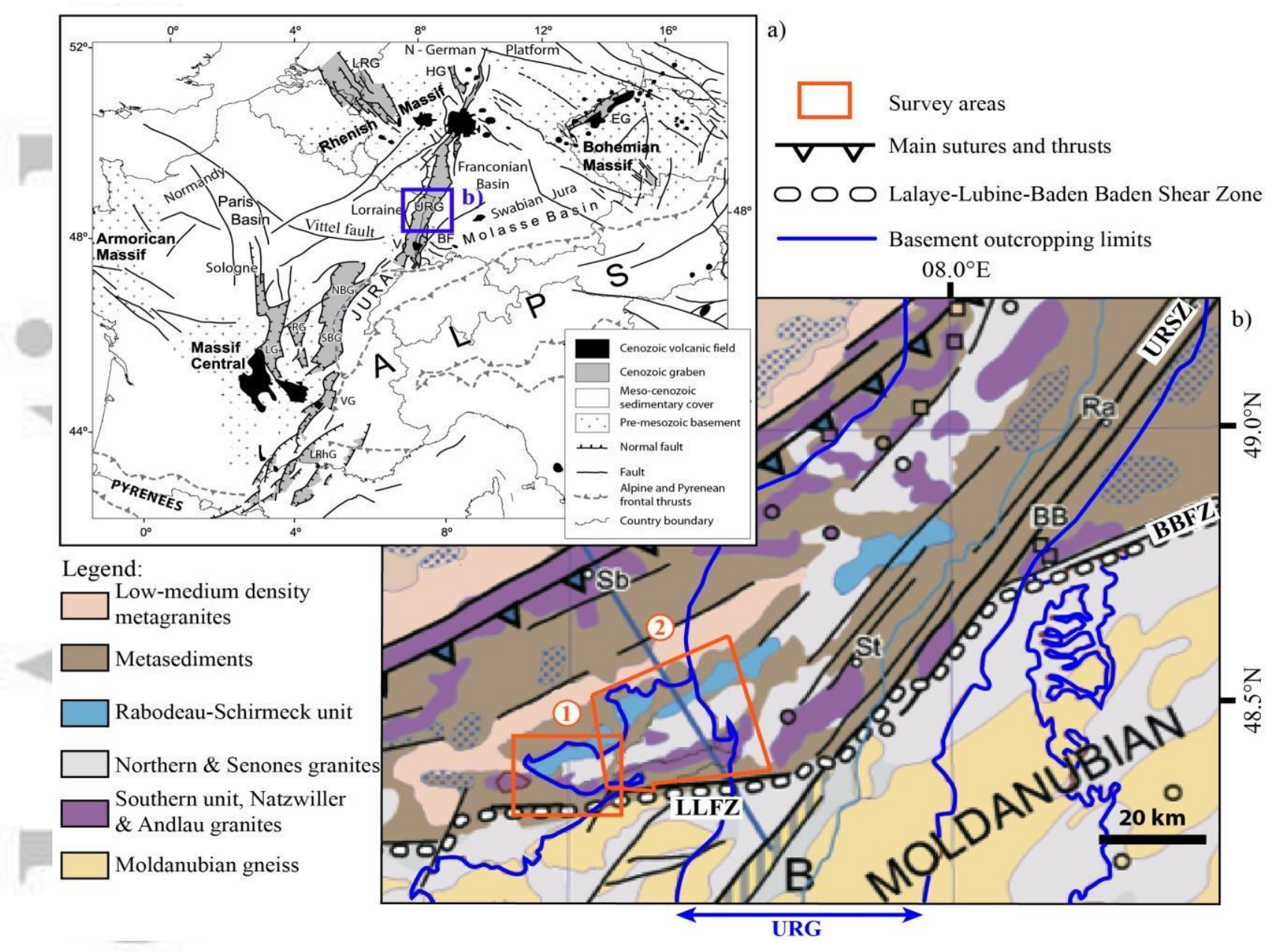

Figure 1. a) General map of the West European Rift System with the associated basins : URG \& LRG : Upper \& Lower Rhine Graben, NBG \& SBG : Northern \& Southern Bresse Graben, RG : Roanne Graben, VG : Valance Graben, LRhG : Lower Rhône Grabens, HG : Hessian grabens, EG : Eger Graben V : Vosges and BF : Black Forest (Bourgeois et al., 2007), b) Interpretative map of the basement rock units from density and gravimetric data within the studied areas, LLFZ : Lalaye-Lubine Fault Zone, BBFZ : Baden-Baden Fault Zone, URSZ : Upper Rhine Shear Zone, St : Strasbourg, Ra : Karlsruhe, Sb : Saarbrucken, BB : Baden-Baden modified after Edel and Schulmann (2009) ; survey 1 is the fixed altitude survey and survey 2 is the survey draping topography. 

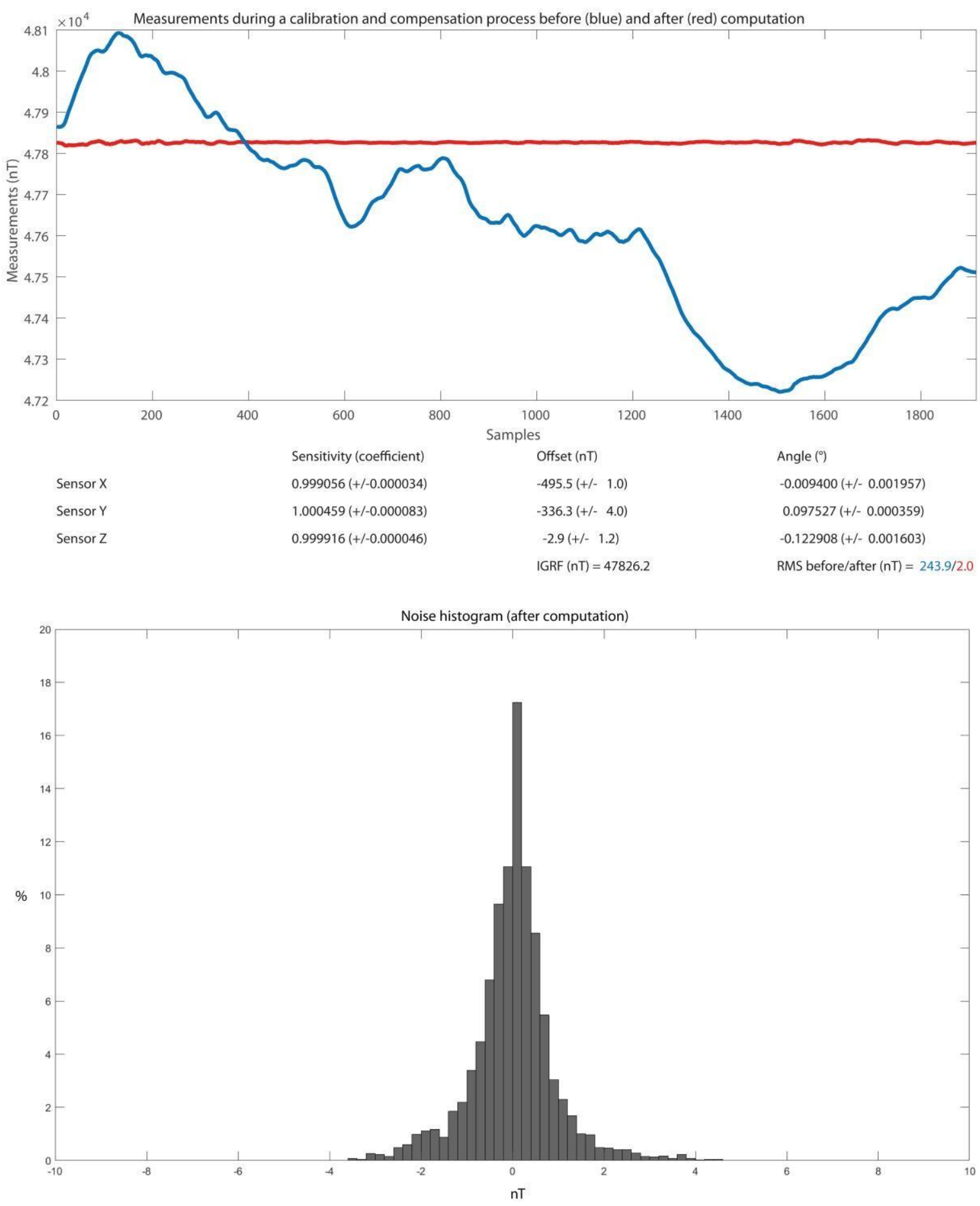

Figure 2. Results of a calibration and compensation process computed from data of the fixed altitude survey. Top: raw data (blue), correction parameters and corrected data (red). Bottom: noise histogram of the calibrated and compensated data. 


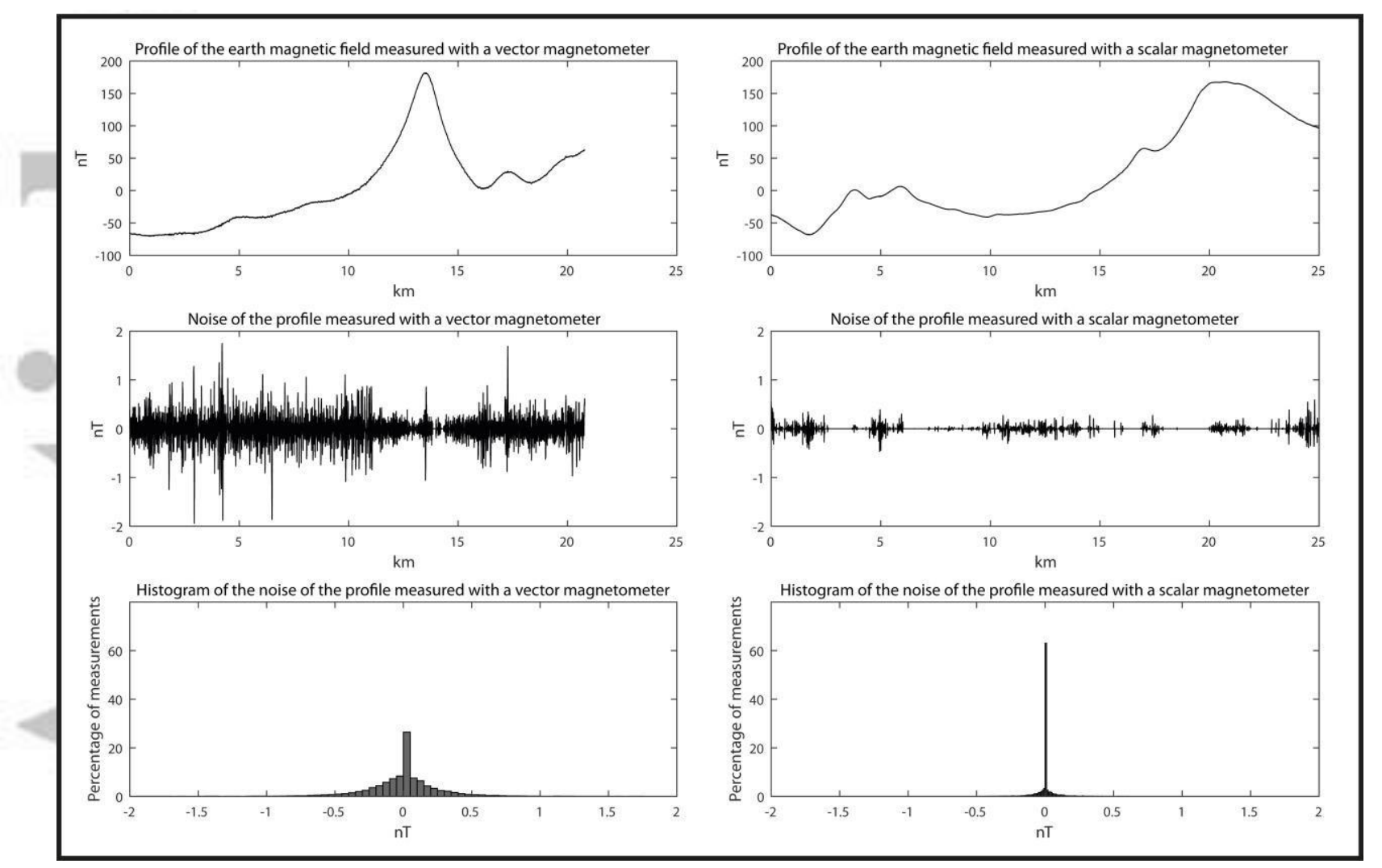

Figure 3. Comparison of the data noise measured with vector and scalar magnetometers 

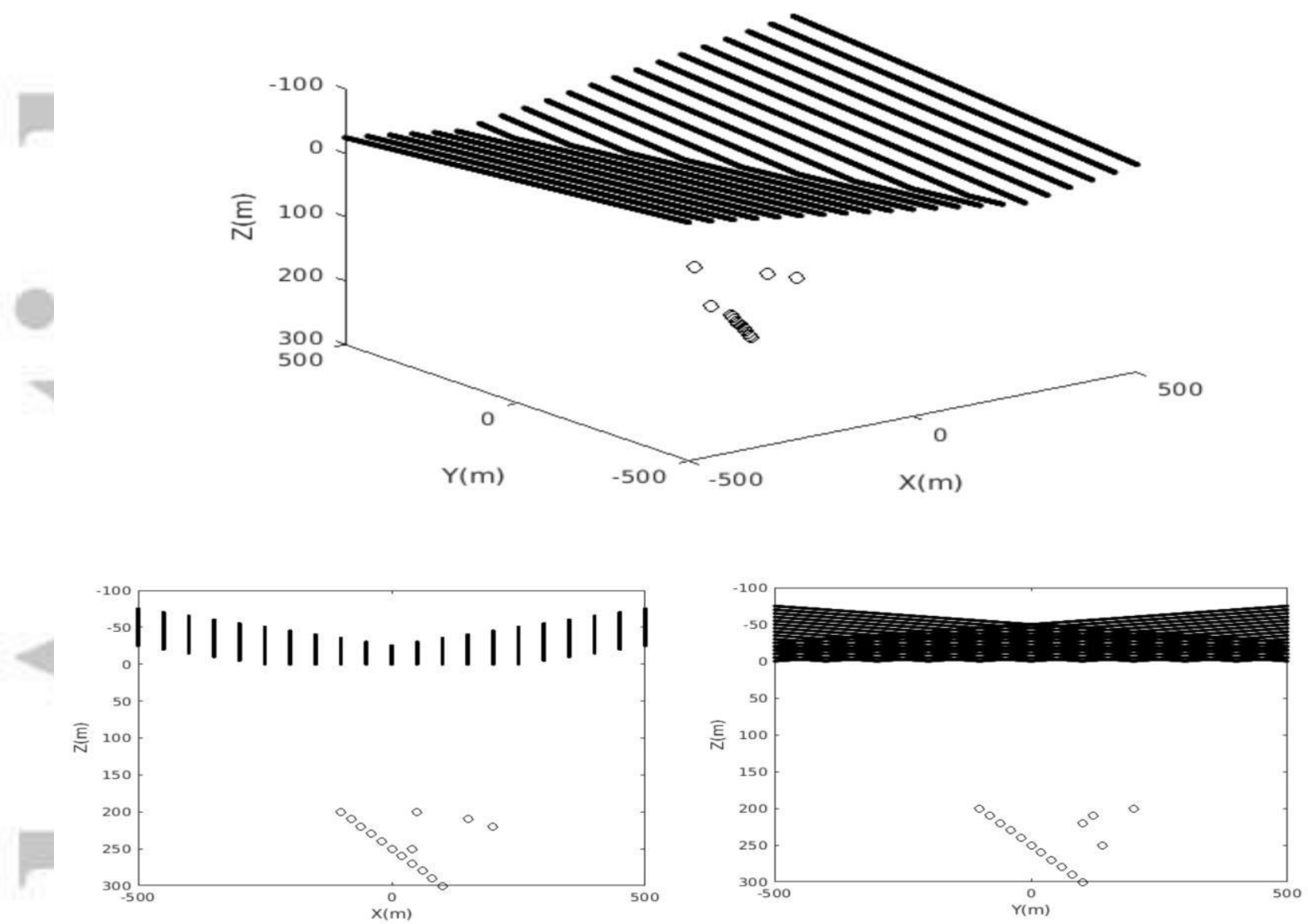

Figure 4. Three dimensional plot of the synthetic model. Synthetic sources are represented with circles and data points with dots forming parallel lines. 


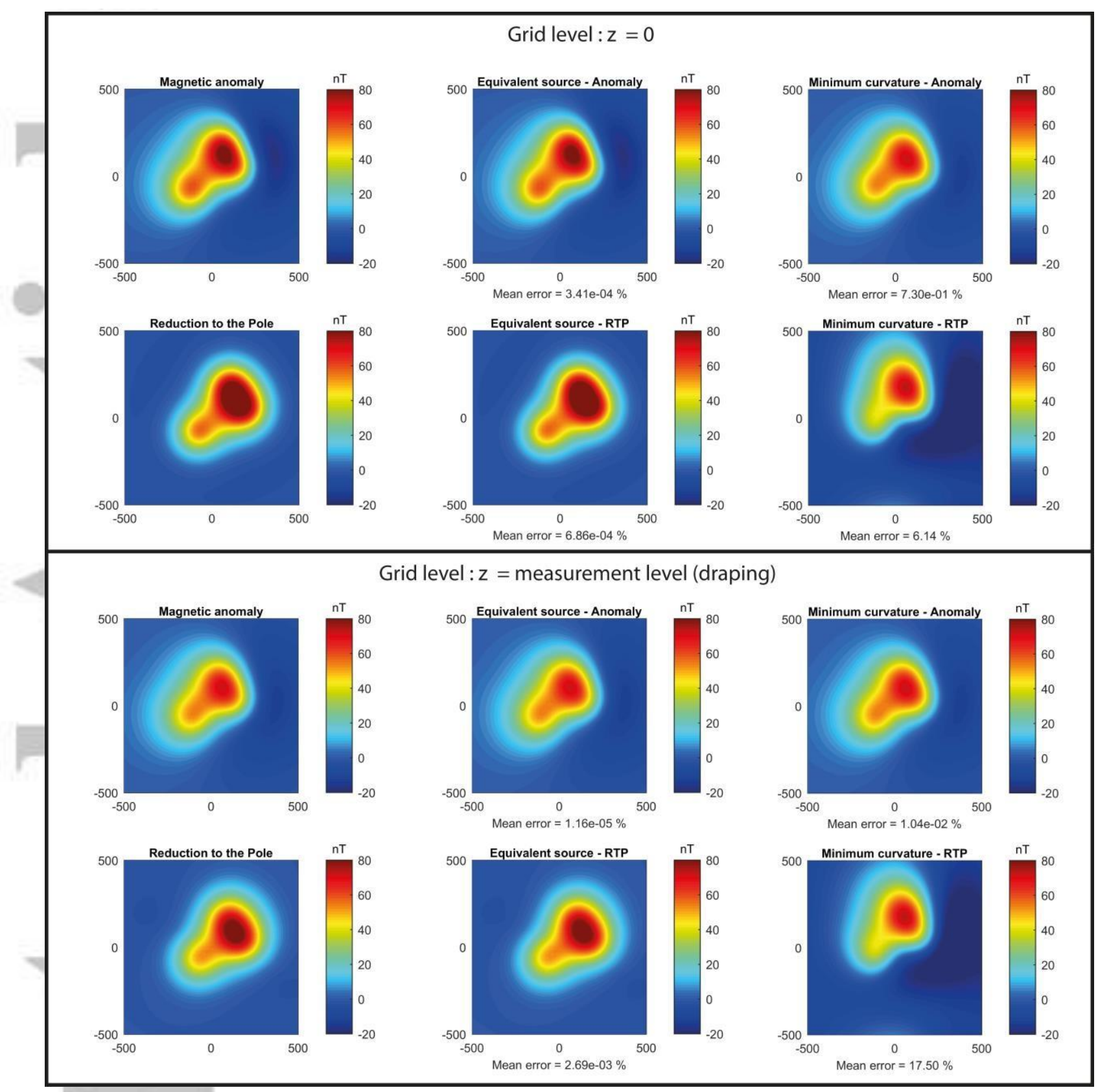

Figure 5. Comparison between the 'true' magnetic anomaly and reduction to the pole (RTP) obtained by direct modeling from the synthetic model and results of equivalent source and minimum curvature gridding methods. Mean errors between results and models are indicated. Top and bottom correspond respectively to a model at a fixed level $(0 \mathrm{~m})$ and draping the varying flight level. 


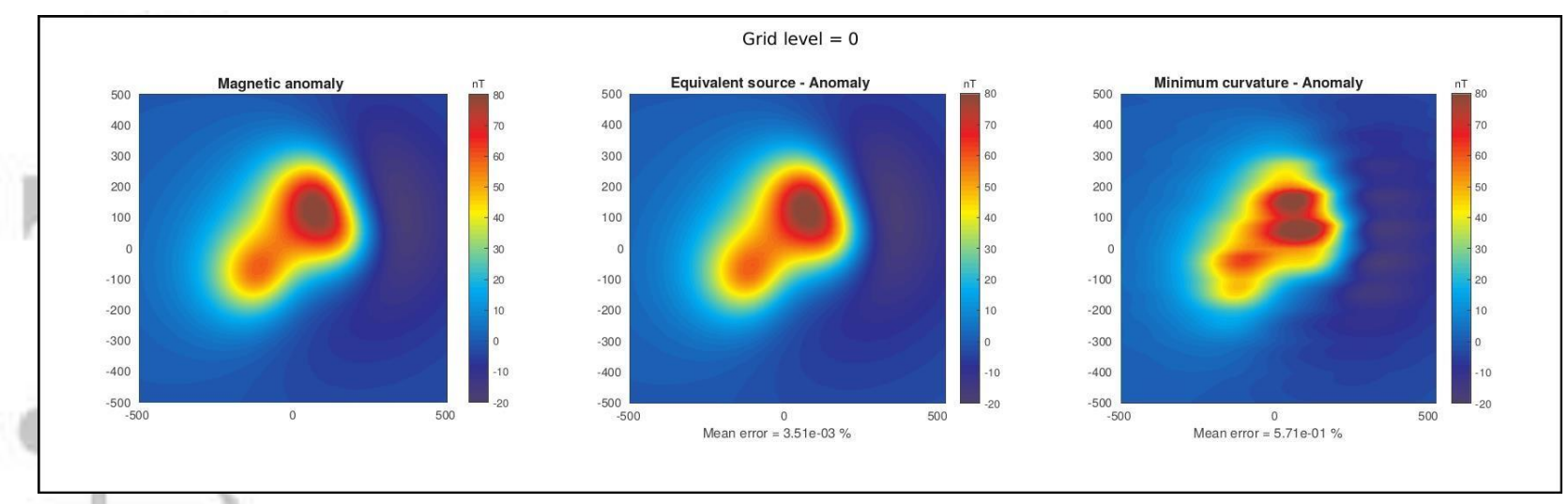

Figure 6. Comparison between the 'true' magnetic anomaly obtained by direct modeling from the synthetic model and results of equivalent source and minimum curvature gridding methods at a fixed level $(0 \mathrm{~m})$ from a dataset with noise. Mean errors between results and models are indicated.

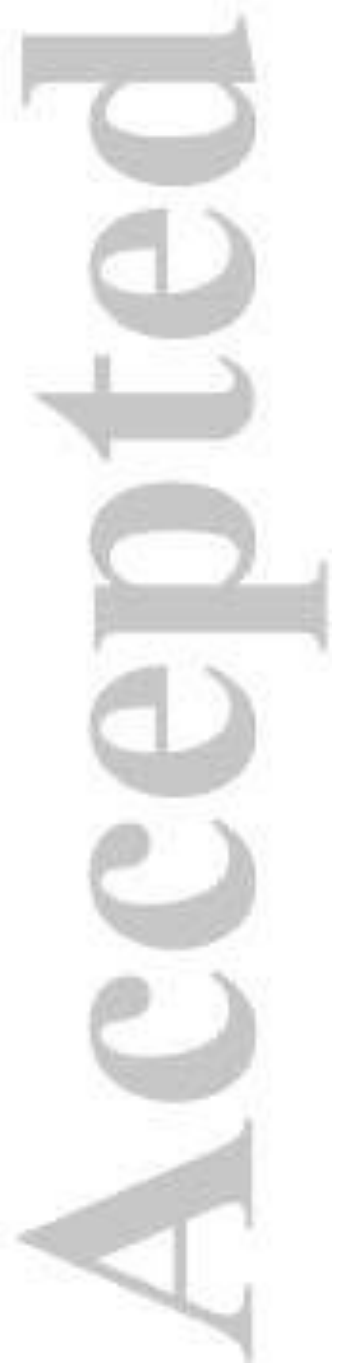




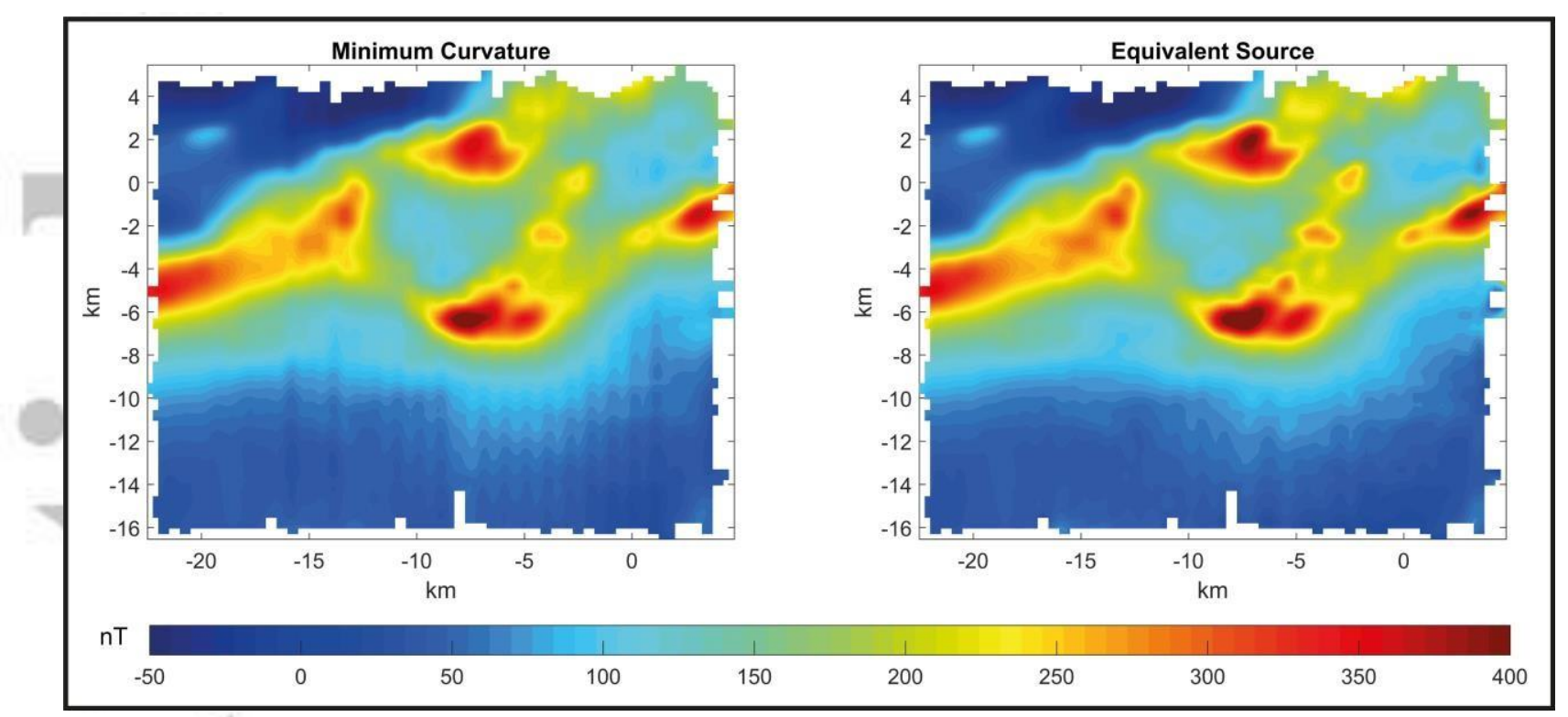

Figure 7. Comparison of anomaly maps of total magnetic field intensity from the levelled survey obtained through the minimum curvature and equivalent source gridding methods.

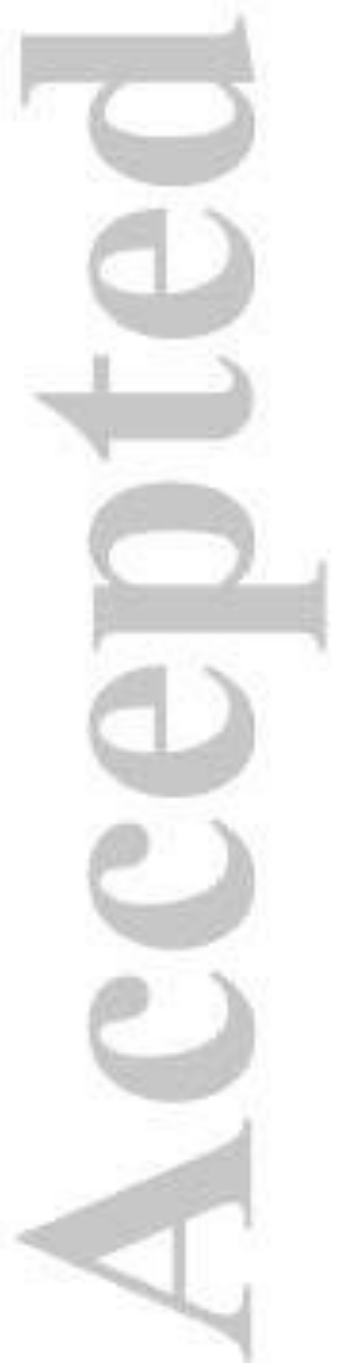




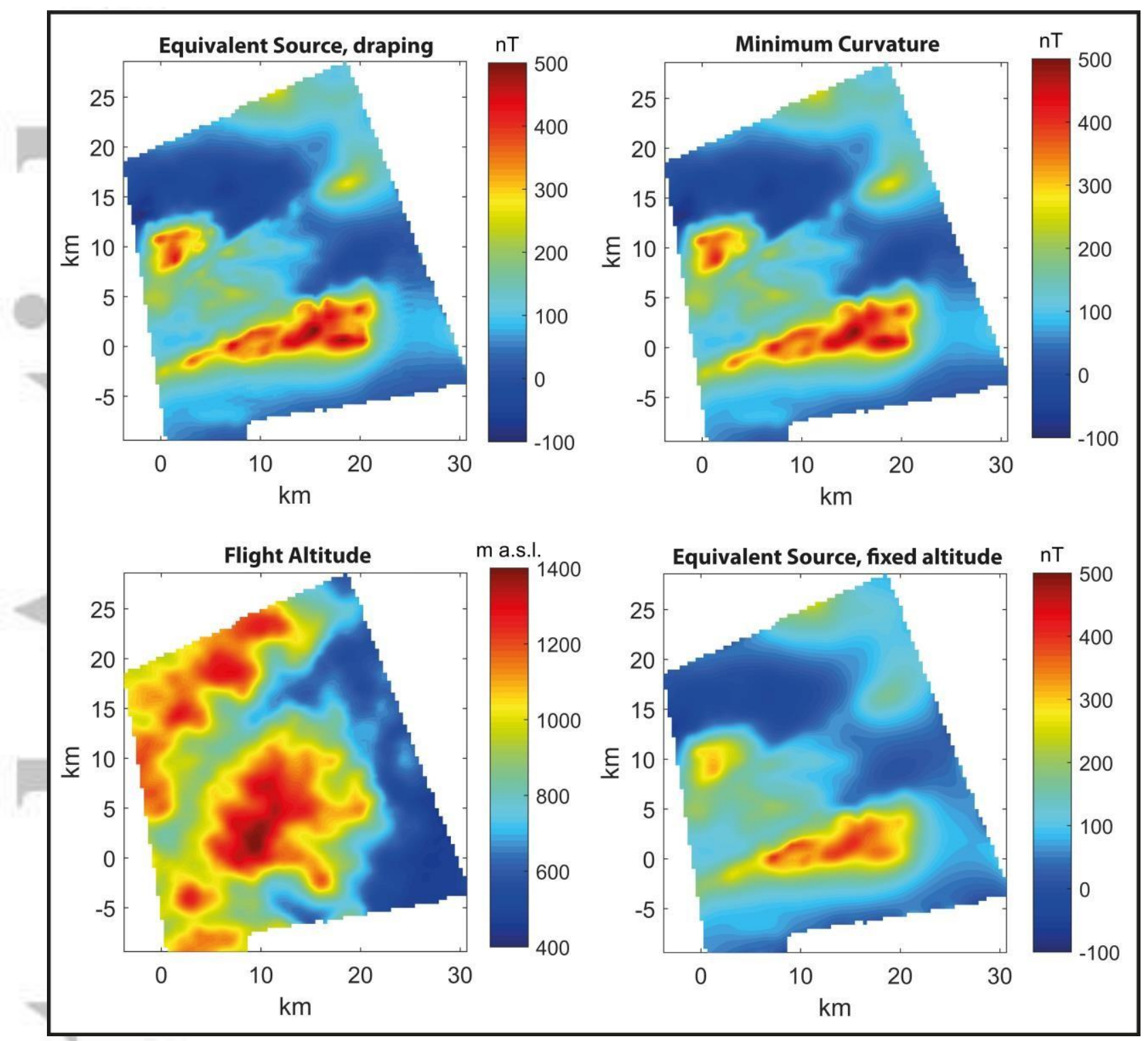

Figure 8. Comparison of maps of the anomaly of total magnetic field intensity from the draping survey obtained through minimum curvature and equivalent source gridding methods. Top left: grid computed at the flight level from equivalent sources. Top right: grid computed with a minimum curvature method modified from D'Errico (2006). Bottom left: flight altitude of the survey. Bottom right: grid computed at a fixed altitude of $1400 \mathrm{~m}$ a.s.l from equivalent sources. 


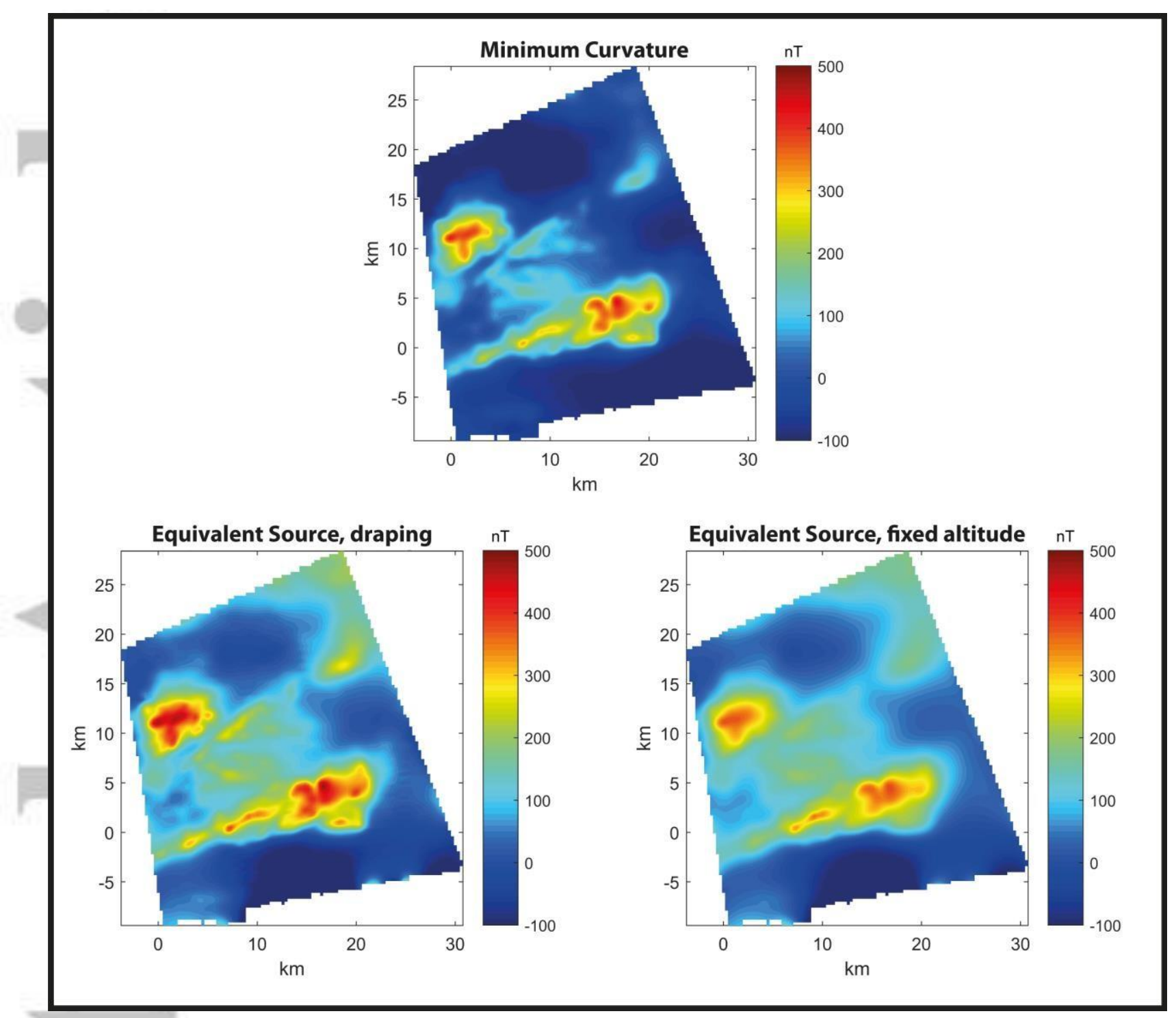

Figure 9. Comparison of maps of the reduction to the pole (RTP) of the anomaly of the total intensity of the magnetic field of the draping survey. Top - RTP computed from a grid obtained through a standard minimum curvature process. Bottom left - RTP computed on a grid draping the topography using equivalent sources. Bottom right - RTP computed on a flat grid at an altitude of $1400 \mathrm{~m}$ using equivalent sources. 


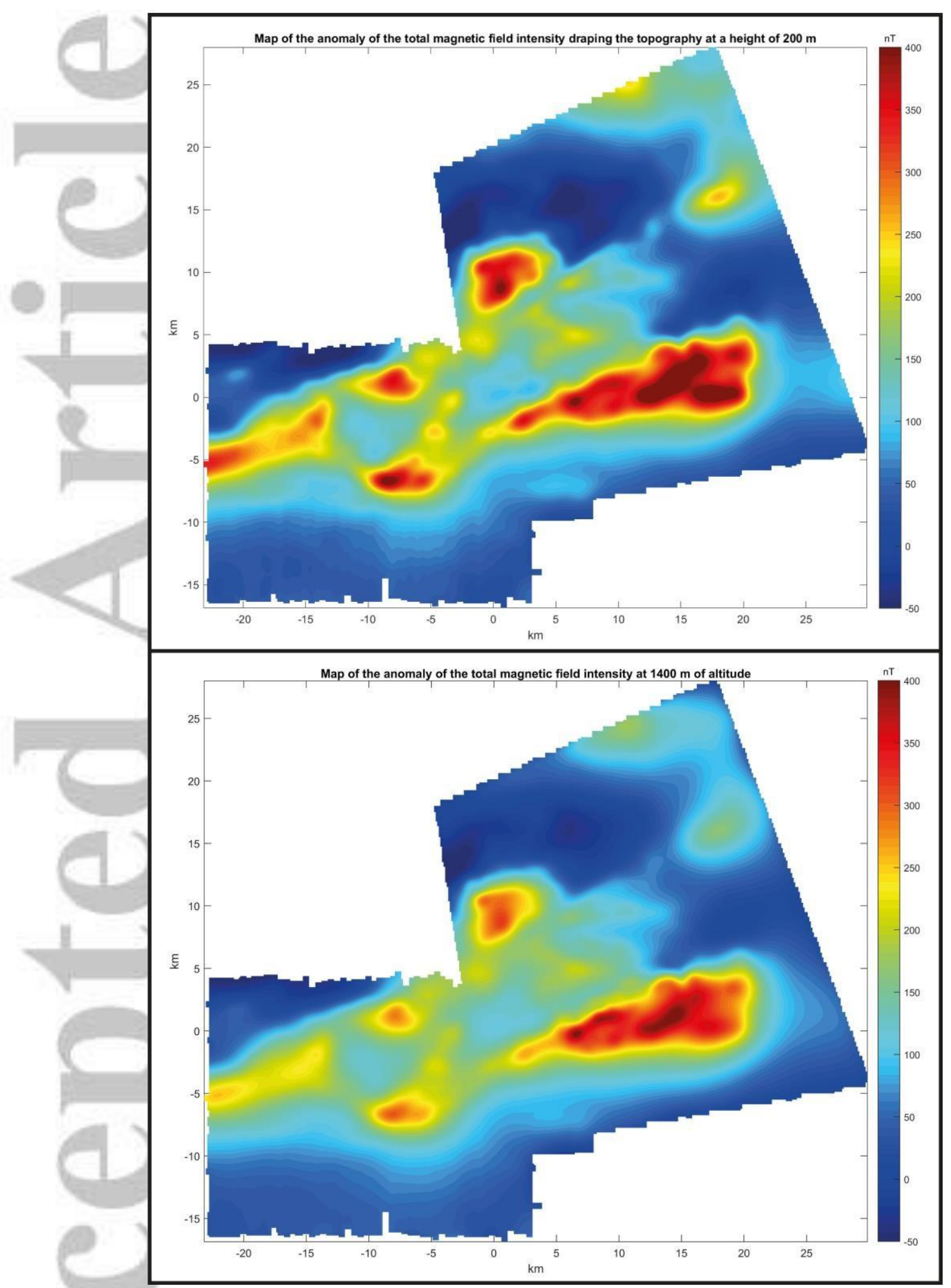

Figure 10. Map of the anomaly of the total magnetic field intensity on a grid draping the topography at a height of $200 \mathrm{~m}$ and a flat grid at $1400 \mathrm{~m}$ of altitude computed by equivalent source method from both surveys. 